\title{
A Physiology-Inspired Multifactorial Toolbox in Soft-to-Hard Musculoskeletal Interface Tissue Engineering
}

Isabel Calejo, ${ }^{1,2,4, @ ~ R a q u e l ~ C o s t a-A l m e i d a ~} \mathbb{1}^{1,1^{1,2,4, @}}{ }^{\text {Rui L. Reis, }}{ }^{1,2,3, @}$ and Manuela E. Gomes ${ }^{1,2,3, *, @ ~}$

Musculoskeletal diseases are increasing the prevalence of physical disability worldwide. Within the body, musculoskeletal soft and hard tissues integrate through specific multitissue transitions, allowing for body movements. Owing to their unique compositional and structural gradients, injuries challenge the native interfaces and tissue regeneration is unlikely to occur. Tissue engineering strategies are emerging to emulate the physiological environment of soft-tohard tissue interfaces. Advances in biomaterial design enable control over biophysical parameters, but biomaterials alone are not sufficient to provide adequate support and guide transplanted cells. Therefore, biological, biophysical, and biochemical tools can be integrated into a multifactorial toolbox, steering prospective advances toward engineering clinically relevant soft-to-hard tissue interfaces.

\section{Musculoskeletal Interfaces and Regeneration Requirements: A Global Burden}

Body tissues and organs are inherently composed of multiple tissues interfacing each other and allowing extremely complex biological functions to take place. In the musculoskeletal system, these tissue interfaces integrate extremely dissimilar tissues with distinctive characteristics, ranging from a hard and highly vascularized tissue with lightweight stiffness and strength, such as the bone, to extremely viscoelastic and avascular tissues, such as articular cartilage, or to tough, resilient, and elastic tissue, such as the tendons (Box 1). Interestingly, the defining characteristic of musculoskeletal interfaces is their primary load-bearing function while mediating the multiple transition in tissues stiffness [1,2], which structurally requires the presence of hierarchically assembled proteins in highly specialized extracellular matrices (Box 1). Examples of soft-to-hard tissue interfaces are the tendon/ligament-to-bone interface, commonly found in the rotator cuff and anterior cruciate ligament, and osteochondral unit (cartilage-to-bone interface) found in the knee joints. However, these tissue interfaces are commonly affected by diseases and disorders at all stages of life. Strikingly, musculoskeletal diseases have been estimated to correspond to the major cause of disability worldwide, with significant healthcare and social support costs [3]. Rotator cuff and anterior cruciate ligament tears or detachment affect the daily life of both adults and the elderly, resulting in pain and movement impairment [4]. Similarly, in adulthood, osteoarthritis, a degenerative process resulting in progressive articular cartilage and joint destruction, especially at the osteochondral interface, is one of the major contributors to immobility, pain, and productivity loss [3]. Current strategies used in the clinics to manage ligament/tendon injuries include the application of grafts (see Glossary) (auto-, allo-, and synthetic grafts) and, alternatively, microfracture surgery, where subchondral bone is perforated to enable a localized transport of bone marrow and blood into contact with the degenerate tissue in the osteochondral unit. However, unsatisfactory functional outcomes of repaired tissues due to previous degeneration, together with increased risk of re-injury, lay the foundation for the development of soft-to-hard

\section{Highlights}

Soft-to-hard interfaces exhibit unique compositional and structural gradients that are difficult to heal and have limited regenerative abilities.

From a biomimetic perspective, combining biological, biophysical, and biochemical cues is likely to enable the generation of physiologically relevant tissue engineered constructs emulating such complex multitissue transitions.

Progresses on cell sheet engineering are integrating cell patterning and mechanical stimulation, together with cells and their matrices as native orchestrators of tissue regeneration.

Advances in biomaterial design offer a precise tuning of architectural, topographical, and mechanical properties to recreate cell-specific niches.

Together with gradients of biochemical cues (including oxygen and growth factors), multifactorial strategies allow strategic control of stem cell differentiation along a single unit.

${ }^{1} 3 \mathrm{~B}$ 's Research Group, I3Bs - Research Institute on Biomaterials, Biodegradables and Biomimetics, University of Minho, Headquarters of the European Institute of Excellence on Tissue Engineering and Regenerative Medicine, AvePark, Parque de Ciência e Tecnologia, Zona Industrial da Gandra, 4805-017 Barco, Guimarães, Portugal

${ }^{2}$ ICVS/3B's-PT Government Associate Laboratory, Braga/Guimarães, Portugal ${ }^{3}$ The Discoveries Centre for Regenerative and Precision Medicine, Headquarters at University of Minho, Avepark, 4805-017 Barco, Guimarães, Portugal 
interface tissue engineering and regenerative therapies. Alternative strategies reaching the stage of commercialization or clinical trials for managing osteochondral defects and tendon-to-bone injuries mainly encompass the application of biomaterials, including biologic and synthetic scaffolds, injectable hydrogels, or decellularized matrices [5,6]. However, these are often used as tissue substitutes, not properly resembling the native architecture, structure, composition, and, therefore, mechanical needs of the native tissue, as well as lacking the ability to generate the proper interface. Hence, the regeneration of soft-to-hard tissue interfaces after injury is still far from being achieved and constitutes a global clinical and economic burden.

The field of tissue engineering and regenerative medicine (TERM) envisions the generation of novel approaches toward restoring functionality upon tissue repair. The ideal strategy for softto-hard tissue regeneration would recreate interfacial physiological complexity, assure mechanical performance of the tissue during the reparative process, and deliver therapeutic elements essential to promote tissue regeneration and to modulate the inflammatory milieu. Besides these challenges, healing must occur within an optimal temporal window toward limiting scar tissue formation. Several studies addressed the role of biological actors, including regenerative competent cells and inflammatory cells, as well as biochemical factors, namely cytokines and growth factors. Alternatively, recent progresses on biomaterial-guided cell behaviors [7] have been providing novel tools to direct cell fate. In this review, we discuss the use of biophysical, biochemical, and biomechanical cues together as a toolbox, steering prospective advances toward engineering physiological niches aimed at soft-to-hard tissue interface regeneration (Figure 1, Key Figure).

\section{A Multifactorial Toolbox for Designing Tissue Engineering Strategies}

Based on the hierarchical organization of soft-to-hard tissue interfaces, various biomaterialsbased approaches have been proposed over the past decade. For tendon/ligament-bone interface, scaffold design has long relied on the creation of stratified layers with or without minerals and reconstructed graft materials for interface repair [8]; however, this does not truly recreate the physiological structure. Therefore, multiphasic and gradient fiber-based scaffold designs, along with strategic patterning of key biochemical cues, such as growth factors, have emerged to emulate multitissue architecture $[9,10]$ while controlling stem cell distribution and differentiation both in vitro and in vivo [11,12]. Comparably, biomaterials-based strategies for osteochondral defects have relied on the use of multilayered polymeric scaffolds, which are designed to generate structural templates to mimic the cartilaginous layer, the calcified cartilage, and subchondral bone, and metallic scaffolds with architectural and biochemical gradients [13,14]. However, these structures do not functionally generate different cellular phenotypes in a spatially and temporally defined way that mimics the native osteochondral tissue cellular environment. Furthermore, in the case of laminate composites, the weak strength bonding between layers normally results in phase separation, not leading to tissue regeneration. Therefore, some reports focused on the use of single scaffolds for osteochondral regeneration $[15,16]$. These scaffolds ensure continuity between phases to avoid a barrier in the interface, together with biomaterials integrity, while mimicking the natural hierarchical structure of the osteochondral tissue. Developed functional materials focused on mimicking specific anisotropic orientation of the subchondral bone and calcified cartilage region in single 3D constructs, demonstrating a favorable control of stem cell fate toward osteogenic and chondrogenic-lineages $[17,18]$. Cartilage-to-bone engineered strategies mainly rely on scaffold-based approaches due to the tunability and versatility of materials to achieve the mechanical and physical requirements of the tissue for proper regeneration. However, developed technologies still fail to provide the necessary signals in a controllable and precise manner for an enhanced and effective biological response, therefore failing in the conjugation with cellular-based strategies.
${ }^{4}$ These authors contributed equally to this work

\section{${ }^{\star}$ Correspondence:}

megomes@i3bs.uminho.pt

(M.E. Gomes).

URL: http://www.3bs.uminho.pt

@Twitter: @3bsuminho (3B's Research Group), @calejo_isabel (I. Calejo), @raquelccalmeida (R. Costa-Almeida), @RLGReis (R.L. Reis), and @ManuelaEGomes (M.E. Gomes). 
Box 1. Overview on Soft-to-Hard Tissue Interfaces Physiology

In tendon-to-bone interface, fibrocartilage connects the aligned, fibrous tendon to the stiff and calcified bone (Figure I). This tissue is characterized by the presence of a nonmineralized and mineralized region, where normally an abrupt transition from cartilaginous to calcified fibrocartilage, often called a tidemark, is observed. This region is also characterized by a gradient of collagen fiber distribution, with an increase in fiber dispersion as it goes far from tendon midsubstance, and a transition between type I and type II collagen [10]. Several studies also demonstrated variations in cellular composition, gene expression, and mineral content along the length of the insertion. Tendon-derived cells, including tenocytes and tendon stem/progenitor cells, are found within tendon, while the fibrocartilaginous interface is populated by fibrochondrocytes and hypertrophic chondrocytes, in the nonmineralized and mineralized regions, respectively. At gene level, data analysis of enriched cartilage and interface transcriptomes suggested that cells found at the interface are more chondrocyte-like than tenocyte-like and that these cells within interface derive from chondrocytes [77]. Also, fibrocartilage at the enthesis endorses a gradual change of elastic modulus, smoothing the transfer of load across the soft-hard tissue interface and therefore resisting compression/shear stress [78].

Comparably, the osteochondral unit is characterized by a chondro-osseous continuum, interfaced by a tidemark, a calcified cartilage, and the subchondral bone plate. Articular cartilage is characterized by a type ll collagen fibrous network interdigitated by sulfate-rich proteoglycans. Beyond the middle zone, these collagen networks are perpendicularly oriented toward the subchondral bone, where an increase in mineralization is extended from the tidemark into the bony counterpart [79]. Evidence suggests a possible direct communication, forming a functional unit, both at mechanical and biochemical levels, with an important role in joint homeostasis. For example, the presence of holes dipping through the calcified cartilage into the bone and marrow spaces and the dense vasculature from subchondral bone found in close proximity with cartilage potentiates the diffusion of nutrients and small molecules between these tissues $[80,81]$. Chondrocytes are the only cell type residing in adult cartilage, owing low metabolic activity and surviving in a hypoxic environment, in contrast with bone cells residing in the subchondral region. Interestingly, subchondral bone cells seem to influence the loss/degradation of cartilage proteoglycans through the secretion of local factors and, in turn, factors secreted from chondrocytes lead to subchondral bone remodeling [82].

Tendon

Fibrils: collagen type I

(A)

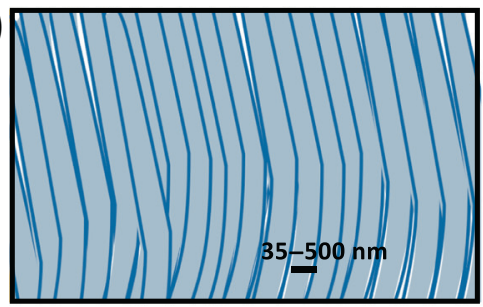

(B)

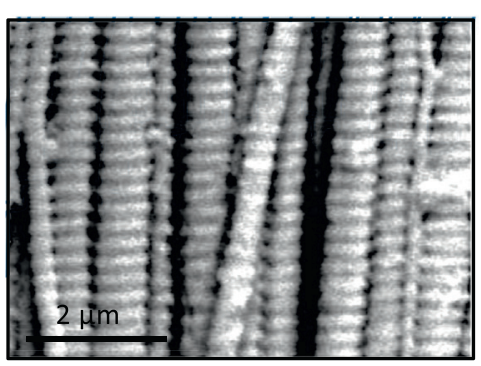

Bone

Fibrils: collagen type I

(C)

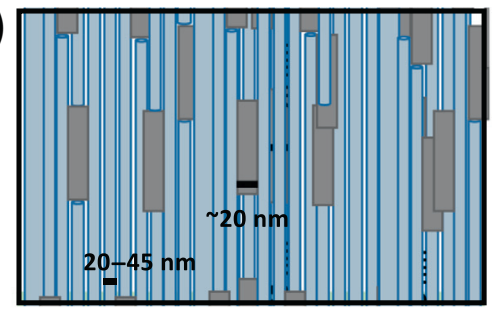

(D)

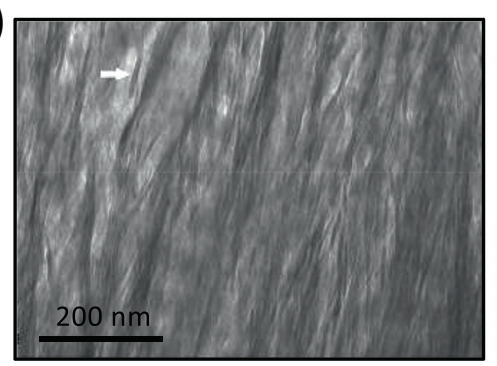

\section{Cartilage}

(E)

Aligned to random mesh: collagen type II

(F)
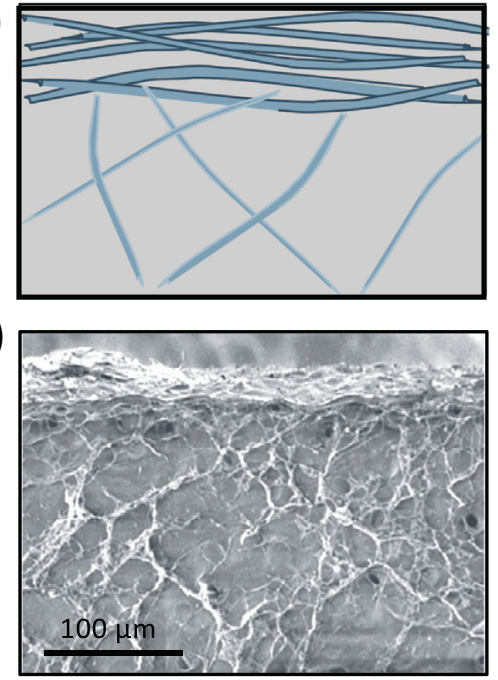

Trends in Biotechnology

Figure I. Matrix Organization of Soft-to-Hard Tissue Interfaces. A collagen rich composition is observed in musculoskeletal tissues, although with different architectural assembly. (A,B) Tendon presents unidirectional collagen type I fibrils (average size, 35-500 nm, blue) arranged in bundles, coupled together in fascicles and these make up the tendon unit. (B) Scale bar, $2 \mu \mathrm{m}$. Reproduced, with permission, from [76]. (C,D) In contrast, bone is constituted by a type I collagenous matrix comprised into stacked fibrils with diameters between $20-45 \mathrm{~nm}$ (blue), which are intercepted by mineral spindles of hydroxyapatite (average size $\sim 20 \mathrm{~nm}$, grey). (D) Scale bar, $200 \mathrm{~nm}$. Reproduced, with permission, from [77]. (E,F) Mature cartilage owns depth-dependent architecture where collagen type II fibers are unidirectionally oriented at the superficial layer, while with depth it the formation of randomly organized meshes is observed. (F) Scale bar, $100 \mu \mathrm{m}$. Reproduced, with permission, from [78].

From a biomimetic perspective, the recapitulation of multitissue spatial properties goes beyond the application of a single strategy. Engineering a functional soft-to-hard tissue interface requires a multiscale, multicomponent approach to integrate region-specific cell populations, matrix composition and organization and, consequently, mechanical requirements in a single unit. 


\section{Biological Tools}

Upon injury, hypovascular dense regular connective tissues (e.g., meniscus, tendons, and ligaments) have a limited self-repair ability and depend on resident or neighboring cells to orchestrate tissue repair, as opposed to the majority of tissues that are supplied by a vascular network, benefiting from circulating progenitor cells. To date, the identification of regeneration competent cells within soft-to-hard tissue interfaces remains a mystery, particularly for tendon/ligament-to-bone interfaces, since resident cells and their heterogeneity are not fully understood.

Different cell populations, particularly bone marrow-derived mesenchymal stem cells (MSCs), and other cell sources ranging from resident/tissue-specific cells to induced pluripotent stem cells, are explored for musculoskeletal tissue regeneration using various delivery strategies (e.g., injection, arthroscopy, implantation), as reviewed elsewhere [19]. Autologous chondrocyte implantation aimed at osteochondral repair was one of the first cell-based tissue engineering interventions to reach clinical application. Similarly, autologous tenocyte implantation is also explored (Phase II-III clinical study NCT01343836). Nonetheless, the need for cell support has been recognized and the combination with matrix-based cell implantation is pursued nowadays.

\section{Scaffold-Free Cell Delivery Technologies}

Cell sheet engineering (Figure 2) has been proposed to overcome shortcomings of single cell suspension injection in tissue reconstruction through preservation of cell-cell contacts and deposited extracellular matrix (ECM) [20]. Autologous cell sheet transplantation has been explored for regenerating thin-layered tissues (e.g., cornea, esophagus, periodontal tissues), but advances in 3D cell sheet manipulation enable the reconstruction of thicker and more complex tissue architectures (Figure 2A-E). Layered chondrocyte sheets alone and in combination with synovial cells or even further combined with scaffolds have been reported to facilitate osteochondral regeneration through barrier functionality and support chondrocyte phenotype and chondrogenic differentiation [21-23]. In the case of tendon and tendon-to-bone repair, the use of native tissue cells and derived cell sheets has been defied by the phenotypic drift and senescence of tendon cells upon in vitro expansion, leading to limited healing capacity. Recently, this has been associated with increased activity of histone deacetylase (HDAC) [24]. Hence, epigenomic modifications targeting the inhibition of different HDAC subtypes allowed the recovery of tendon-marker scleraxis expression, supporting the use of tendon stem/progenitor cells (TSPC) sheets (Figure 2F) in accelerating tendon repair [24]. Further combining stem cell sheets with native tendon-fibrocartilage-bone composite as a biological patch to augment rotator cuff healing resulted in enhanced fibrocartilage formation and collagen fiber organization, while providing biomechanical support during tissue repair [25]. The value of cell sheets in tissue repair is well recognized, as they overcome cell loss and reduced engraftment upon transplantation. Nevertheless, engineering functional soft-to-hard tissue interfaces is postulated to require an integration of physiologically relevant ECM signals and mechanical stimuli toward guiding stem cell fate.

Next-generation cell sheet engineering is taking this technology to a higher level of organization in recreating complex tissues. The development of anisotropic cell sheets using stripe-like micropatterned thermoresponsive surfaces [26] (Figure 2A) and, recently, light-induced cell alignment and cell sheet harvest [27] (Figure 2B-D) hold promising results to be explored for interfacial tissue engineering. More recent trends have been exploring magnetic force-based tissue engineering. The generation of magnetic cell sheets by cellular internalization of magnetic nanoparticles enabled the fabrication of tenogenic living ECM-rich patches (Figure 2E) with potential for remote control upon application of an external magnetic field as mechano-magnetic stimulus [28].

\section{Glossary}

Biochemical cues: molecules involved in chemical reactions within living organisms that have the ability of initiating or modifying a biochemical or signaling cascade; such signals can be mimicked in vitro by culture supplementation or biofunctionalization. Biofunctionalization: modification of a material surface for either specific or nonspecific immobilization of defined motifs or biomolecules that add biological functionality in addition to biocompatibility/tolerability by the body. Biomechanical/biophysical cues: physical signals or forces from the external environment that are perceived through the mechanosensory machinery of cells and activate signaling cascades; such signals can be mimicked in vitro by specific mechanical properties or culturing systems, as well as substrate properties like elasticity, rigidity, and topography

Biomimetic: replication/imitation of biological systems.

Bioreactors: mechanical devices or systems used for providing regulatory biochemical and physical signals to cells, in a dynamic manner, toward enabling stem cell differentiation and/or extracellular matrix deposition and replicating tissue-specific requirements. Cell sheet: confluent cell layer with intact cell-cell junctions and extracellular matrix resulting from the detachment of cell cultures commonly from thermoresponsive surfaces or using alternative approaches (e.g., light-induced, magnetic force).

Engraftment: response given by the body in which cells are accepted after transplantation.

Epigenomic modifications: in vitro manipulation of cellular processes that regulate the transcription of genetic information through pharmacological tools, genetic editing, and precision epigenetic editing.

Extracellular matrix: 3D network of structural macromolecules produced by cells into the surrounding environment, including collagens and other proteins, proteoglycans, and glycoproteins.

Gradient: an increase or decrease in the magnitude of a biochemical/ biophysical property or a variation in cellular content/type.

Graft: a small sample of living or synthetic tissue that is surgically transplanted. 
Biotechnological advances are pushing forward the complexity of cell-based therapies, paving the way to engineer living constructs with physiological and clinical relevance. Despite the promising reported outcomes, unsolved issues remain that challenge the field, including the development of off-the-shelf cellular therapies. Further, the refinement of cellular approaches in combination with biophysical and biochemical tools to manipulate cell fate is a current need toward generating physiologically relevant cellular gradients to emulate the cellular niche from the different musculoskeletal interfaces.

\section{Biophysical Parameters}

Soft-to-hard musculoskeletal interfaces are highly structured nanocomposites arranged into microarchitectures with unique directionalities, gradients, and cellular environments. Attachment of cells to the ECM regulates diverse cellular functions. It is well recognized that a precise control over nano-to-macro structural features of biomaterials is of major importance to recreate key properties of the native ECM. However, the development of multitissue transitions is still a challenge.

Advances in biomaterials design allow for refining microenvironmental properties, paving the way to generate physiologically relevant niches and engineering soft-to-hard tissue interfaces. Over the years, different fabrication methods have enabled control of a panoply of interdependent biophysical parameters (Table 1).

Tailoring Biomaterial Architecture, Surface Topography, and Mechanical Properties

As biophysical properties of native ECM are difficult to emulate, synthetic fibrous scaffolds have been essential to study and regulate specific matrix properties, important for cellular proliferation and function. From electrospinning to hydrogel systems, tendon-to-bone regeneration has been relying on the fabrication of variable scaffolds for the replication of architectural and mechanical properties of the graded tissue. Nanofibrous scaffolds have been widely used for tendon-tobone regeneration as these biomaterials act as a physical platform mimicking 3D fibrous collagenous hierarchical structure [29,30]. In contrast, 3D printing and hydrogels have been used for mimicking physical properties of the osteochondral unit, as this combination demonstrates good physical and mechanical performance [12,31].

The development of injectable hydrogel systems with control over the architecture of the fibrillar network has been also demonstrated to have biological relevance [32]. These hydrogels have a controllable and precise internal fibrous structure, which determines their pore size and mechanical properties, while replicating the filamentous architecture of the ECM. Moreover, hydrogels can also be tuned to present an anisotropic architecture, for instance, taking advantage of magnetic stimulation to nanoparticle alignment [33]. Strikingly, these hydrogels provide biochemical and physical cues, enabling us to tune the behavior of encapsulated stem cells, with prospective applications in minimally invasive defect filling surgeries.

Within every tissue and organ, cells sense the properties of their supporting environment at multiple length scales. Hence, new biomaterial designs must consider the role of mechanosensing. By generating methacrylated dextran fibrillar matrices resembling collagen type I networks, Baker and colleagues have demonstrated the role of fibrillar topography in directing cellular morphology, impacting cellular alignment and matrix remodeling by cellular traction forces [34]. Remarkably, cellular forces at the microscale, together with tissue-generated forces at the macroscale, are two essential parameters guiding numerous tissue differentiation and maturation during soft-tohard tissue development [35]. In turn, cellular processes, including stem cell lineage specification, can be guided by contact with the surrounding physical microenvironment. Matrix stiffness as a
Growth factors: naturally occurring molecules, usually proteins, which stimulate cell growth, proliferation, differentiation, survival, and regulate various cellular processes, including tissue homeostasis and healing. Homeostasis: a relatively constant equilibrium in the internal physical and chemical conditions maintained at different levels (cellular, tissue, organ, system) by living organisms through physiological processes.

Hydrogel: crosslinked 3D network of natural or synthetic polymers that can absorb and retain large volumes of water or biological fluids without polymer dissolution.

Hypoxia: condition in which the oxygen supply is lower than the normal arterial blood concentration

Induced pluripotent stem cells: pluripotent stem cells generated from adult somatic cells that were reprogrammed back into their embryonic-like state through the addition of a transcription factor cocktail under embryonic stem cell culture conditions.

Magnetic force-based tissue engineering: tissue engineering strategies that rely on the use of magnetic nanoparticles and external magnetic actuation to direct cell positioning/ behavior.

Matrix stiffness: property of a materia to resist deformation in response to an applied force and defined as the ratio between the applied force and deformation.

Mechanosensing: ability of a cell to feel the mechanical/physical cues of the surrounding environment, including force components, substrate deformation, and properties (see 'Biochemica//biophysical cues'). Multilineage differentiation: potentia of stem cells to develop into a multiple number of cells types.

Nanocomposites: materials in which one of the phases is within the nanometer range $(<10 \mathrm{~nm})$.

Progenitor cells: cells that are typically descendants of stem cells but with more constrained differentiation potential and self-renewal capacity. 


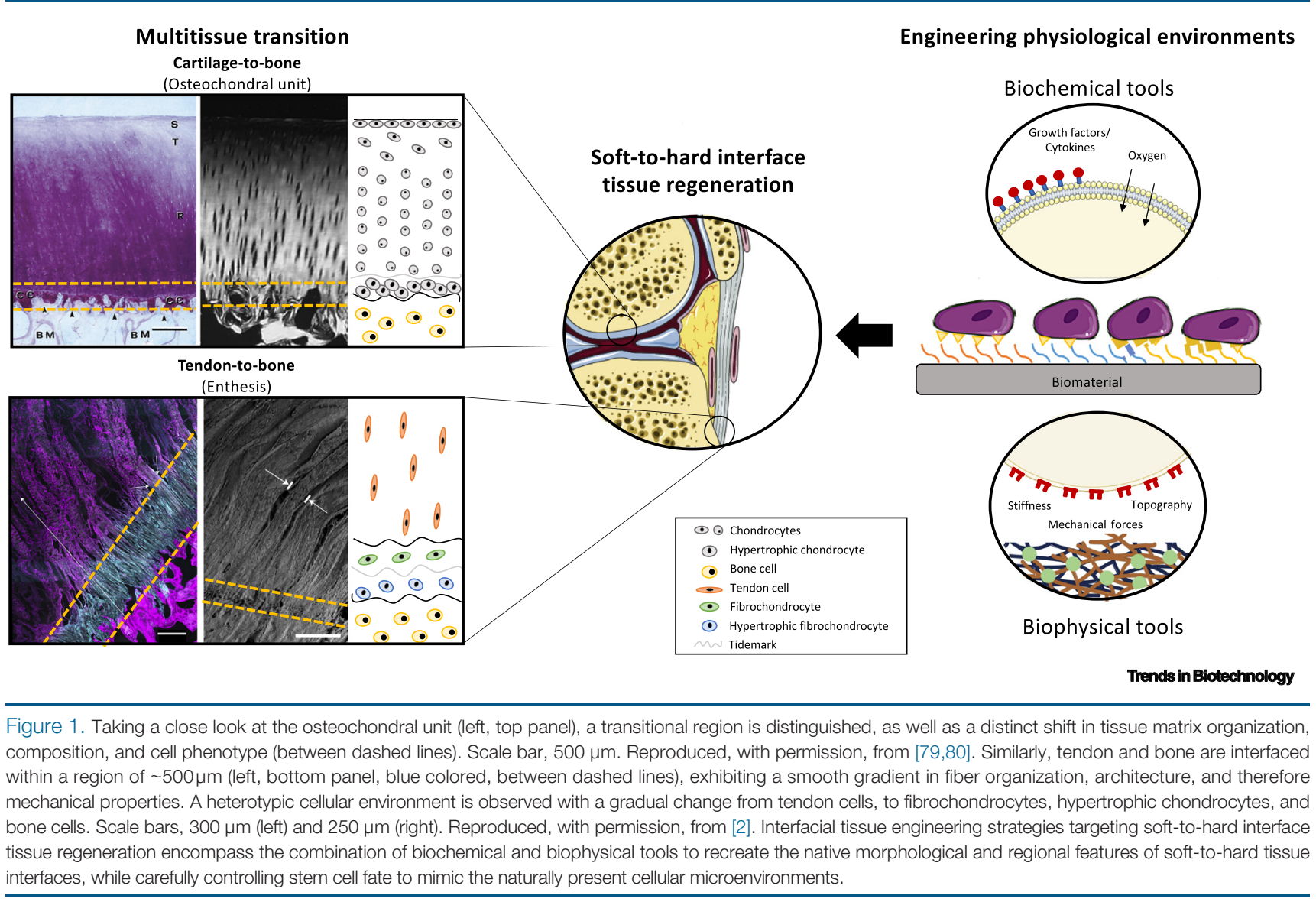

stand-alone stimulus directing multilineage differentiation of bone marrow MSCs was first demonstrated in 2006; for instance, rigid matrices resembling collagenous bone triggered osteogenic differentiation [36]. Surface stiffness was shown to modulate stem cell adhesion, proliferation, and differentiation (Figure 3A). Indeed, MSCs tend to become more spread on substrates with higher stiffness (ranging from 50 to $90 \mathrm{kPa}$ ) and differentiate toward chondrogenic $[37,38]$ and osteogenic lineages $[38,39]$ (Figure $3 \mathrm{~A}, a-b)$, whereas softer surfaces $(\sim 30-50 \mathrm{kPa})$ result in a tenogenic-like phenotype $[39,40]$ (Figure 3A, $c$ ). These results support the creation of material stiffness gradients to guide stem cell differentiation along tendon-to-bone and bone-cartilage bioengineered constructs. Such gradients can be reproduced as a consequence of mineral content in nanofibrous systems, taking advantage of simple mineral coatings to increase nanofibrous stiffness to direct stem cell differentiation toward the osteogenic lineage [41]. Comparably, hydrogel stiffness has been shown to have a role in modulating cell-based osteochondral formation in 3D, as soft hydrogels have been shown to support the deposition of neocartilage by cells due to their permissiveness [42] and stiffer hydrogels have been shown to support the deposition of osteogenic-like matrix [43]. Complementarily, micro/nanostructured surface architectures have been developed as an easy and smart strategy to induce bi-lineage differentiation in single 
scaffolds (Figure 3B). Interestingly, tailoring the surface topography of scaffolds by increasing either the roughness or the use of nano-scaled matrices has been shown to allow a better cell adhesion to the matrix, followed by subsequent tenogenic, osteogenic, and chondrogenic commitment of stem cells when in contact with oriented groove materials or just by creating dense or fibrous topologies in scaffolds, respectively [10,18,44-47].

In this sense, it is worth mentioning that phenotypic alterations induced by biophysical sensing have been shown to have a dose-dependent response and to be cell type-specific and context dependent [48]. It has been long recognized that material properties (e.g., matrix stiffness, topography) can sensitize cells to other microenvironmental features, impacting cell response [49-52]. Notwithstanding, recent studies using RNA sequencing and differential expression analyses have demonstrated that one specific parameter has the power to contextualize the response to other features, through a dose-dependent effect $[48,52]$. Besides this context-dependence of coupling biophysical cues, the type of cell also determines the downstream response and the way the biophysical sensing happens. Indeed, the ability of cells to cluster adhesion ligands in response to a specific material parameter has been demonstrated to occur in a cell type-specific manner and to be dependent on cellular intrinsic characteristics (lineage, species) [48].

These findings raise several questions, particularly concerning the interplay of downstream cell regulatory networks in response to specific combinations of biophysical features. Hence, challenges emerge regarding the establishment of adequate gradient biomaterials for interfacial tissue engineering. Other challenges include the impact on selection of cell sources and cell history for developing adequate tissue mimetics. Biophysical gradients are expected to guide the behavior of different cells types, which will, in turn, establish a cross-communication and influence each other, resulting in a highly complex system.

\section{The Role of Mechanotransduction}

Since the function of musculoskeletal interfaces is to bear and transmit loads between mechanically different tissues, it is not a surprise that mechanical loading contributes to the development and function (and even pathology onset) of such multitissue interfaces, contributing to a gradation in tissue cellularity and structure. During the last few years, growing evidence has demonstrated that cells sense the mechanical forces in different ways, transducing these mechanosignals into gene regulation that will impact not only cell migration or ECM adhesion but also proliferation and differentiation [53]. Therefore, understanding cellular responses upon stimulation by mechanical inputs from the surrounding environment may provide key information for manipulating cellular behaviors toward proregenerative phenotypes.

Active loading has been used in vivo to guide tissue formation upon construct implantation, but issues remain regarding the lack of control over mechanical loading regimes. Alternatively, effects of mechanical stresses have been increasingly explored in vitro using dynamic systems as bioreactors. The potential regeneration of tendon/ligament-bone has been evaluated mainly through the use of cyclic tension, whereas bone-cartilage regeneration has relied on the use of compressive stress. For instance, a dynamic compression bioreactor was used in a semi-confined compression model to direct MSC differentiation throughout the depth of a hydrogel to resemble the spatial endochondral progression [54]. The application of dynamic compression increased strains across the top of the construct, while the confinement reduced oxygen levels (see section 'Biochemical Tools') at the bottom of the construct, resulting in increased glycosaminoglycan accumulation in the bottom, increased collagen accumulation in the top along with a suppression of hypertrophy and calcification throughout the construct [54]. In contrast, mechanical and biological properties of an engineered tendon-to-bone composite have been investigated after culturing 
(A)

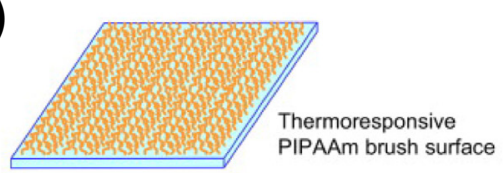

Site-selective grafting
of hydrophilic polymer
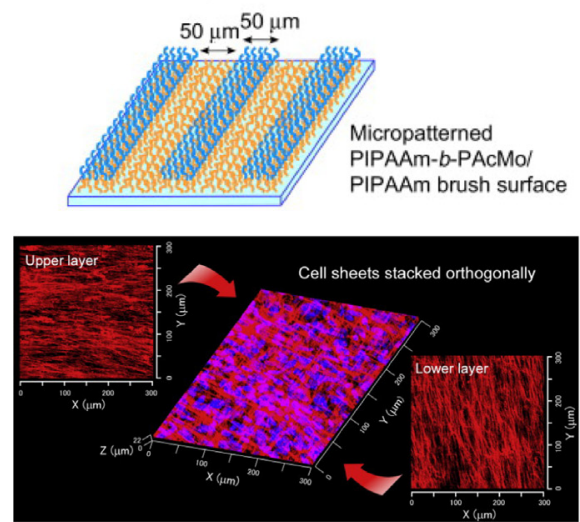

(C)
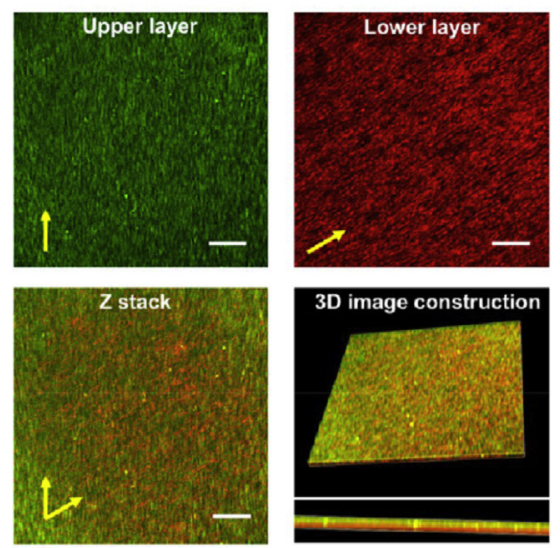

(E)
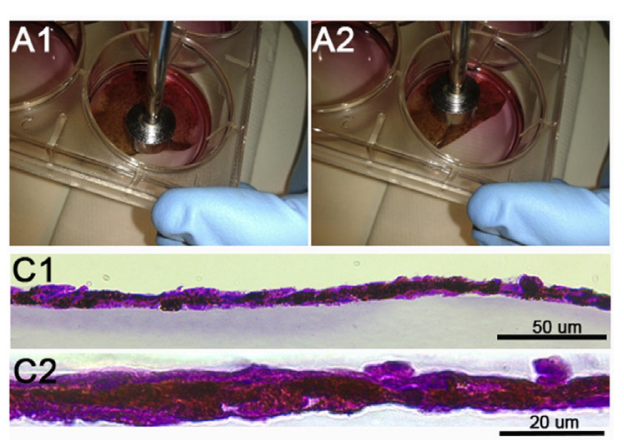

(B) UV-induced cell patterning on $\mathrm{TiO}_{2}$ nanodots film (TNF)

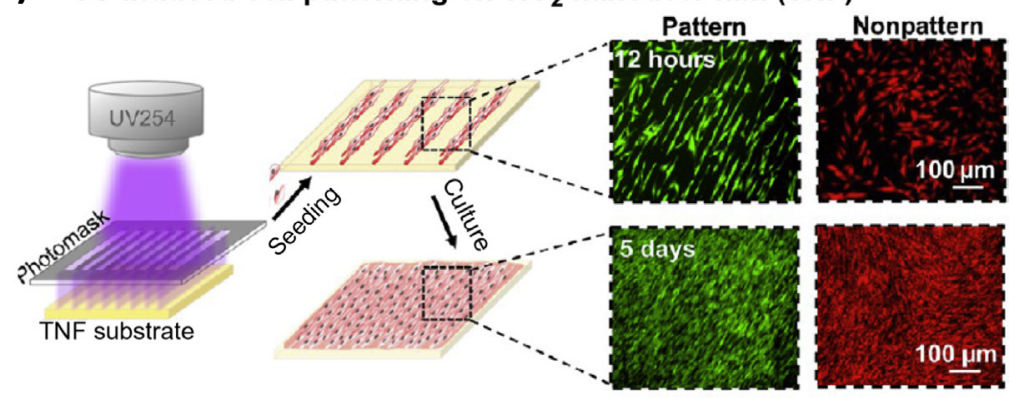

UV-induced anisotropic cell sheet (ACS) detachment on TNF

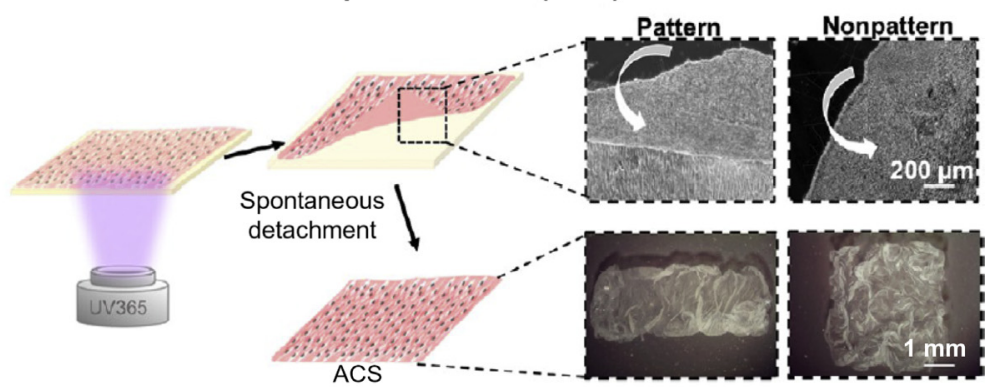

(D)
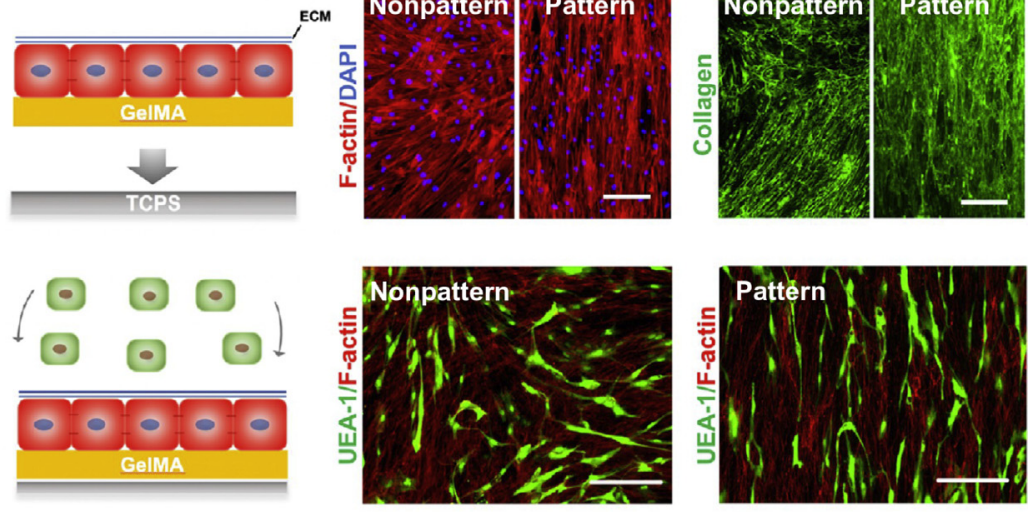

(F)
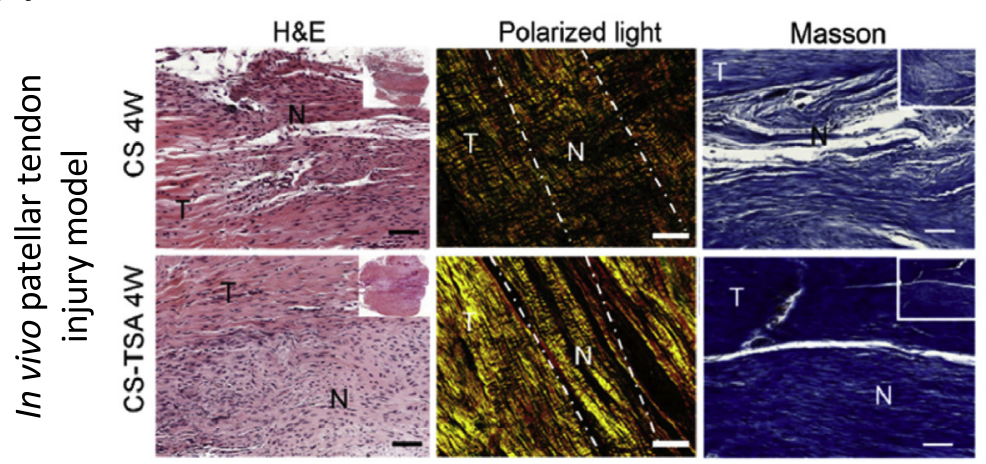

Trends in Biotechnology

(See figure legend at the bottom of the next page.) 
bone marrow-derived MSC sheets under cyclic tension for 7 days [55]. Mechanical tension led to increased cell migration and aligned distribution of cells within the scaffold, resulting in the upregulation of tenogenic genes as scleraxis [55].

While there are some challenges to overcome in engineering a native-like soft-to-hard tissue interface, whether bioreactors systems will be ultimately used for tissue maturation for clinics remains an open question. However, these systems present an advantage over current in vitro systems as they allow fine control over mechanical cues, improving both local cell modulation and, ultimately, tissue regeneration after implantation.

\section{Biochemical Tools}

Supplementation of cell cultures using biomolecules has been explored to maintain the phenotype of permanently differentiated cells or to modulate stem cell fate and induce differentiation. Among different biochemical factors, oxygen is a crucial molecule and we will discuss the influence of oxygen tension on cellular behavior. Additionally, small molecules, like ascorbic acid, can be employed to accelerate ECM deposition, particularly for cell sheet engineering [56-59], or to promote cellular proliferation in cell expansion protocols (e.g., glucose and essential amino acids). Other signals include hormones, growth factors, and cytokines. Although high-throughput analysis of combinatorial approaches has been troublesome, the use of such biochemical cues has been at the forefront of cell-based therapy development and is herein addressed.

\section{Oxygen Tension and Hypoxic Niches}

In the human body, normal physiological conditions of oxygen tension fall between $2 \%$ and $9 \%$ in most organs and between $10 \%$ and $13 \%$ in arteries, lungs, and liver [60]. The musculoskeletal system is a good example of where hypoxia plays an important role during tissue development and homeostasis. Soft-to-hard tissue interfaces present a gradual transition in vascularity and, consequently, in oxygen concentrations. Given the physiology of the native tissues (Box 1), tendon cells are known to maintain their phenotype in vitro under hypoxic conditions, as the ones observed in the native hypovascular tendon niche ( $<5 \%$ oxygen tension) $[61,62]$. Contrarily, hypoxia has been shown to profoundly inhibit osteoclastogenesis in bone remodeling [63] given that bone physiological oxygen tension remains above 5\% [64].

Nonetheless, the beneficial effect of hypoxia has been demonstrated using different sized engineered endochondral cartilage intermediates prior to implantation, where hypoxia activation resulted in improved bone formation and enhanced chondrogenesis without the formation of fibrotic tissue [65]. Moreover, several studies have demonstrated the role of oxygen in directing both cartilage and bone integration, whereby hypoxic conditions $\left(2.5 \% \mathrm{O}_{2}\right)$ have been shown to suppress hypertrophy and endochondral ossification within chondrogenically primed MSCs [66]. Tissue engineering strategies have been addressing the effects of cell conditioning under

Figure 2. Advances in Cellular-Based Technologies. Cell sheet engineering enables the delivery of cells along with their own extracellular matrix. Site-selective polymer grafting onto thermoresponsive surfaces enabled the generation of anisotropic cell sheets upon one-pot cell seeding of human dermal fibroblasts (A). Reproduced, with permission, from [26]. Recently, light-induced cell alignment and harvest was achieved through the combination of light-responsive nanodots and photocrosslinkable gelatin (B). These anisotropic cell sheets retain an aligned extracellular matrix and can be stacked together in several layers to generate more complex 3D constructs (A) top and (C). Besides the control over cell and matrix orientation, anisotropic cell sheets induce endothelial cells seeded on top to orient themselves, favoring vascularization (D). Although not yet applied to soft-to-hard interface tissue engineering, these strategies hold promising features to be manipulated envisioning the creation of a gradient of cells, cellular alignment, and cellular environments. (B-D) Reproduced, with permission, from [27]. Progresses in magnetic force-based tissue engineering are also supporting the development of magnetic cell sheets (magCS). MagCS have been fabricated using a subpopulation of tenomodulin-expressing human adipose tissue derived stem cells (ASCs) to support the development of tendon-like living patches, which can be harvested through the use of a permanent magnet (E). Reproduced, with permission, from [28]. Further manipulation of tendon stem/progenitor cells through epigenetics tools [cell sheets treated with trichostatin A to inhibit histone deacetylase (CS-TSA)] allowed long-term cell expansion until a sufficient cell number was reached for cellular therapies with in vivo evidence of therapeutic efficiency in a rat patellar tendon injury model (F). Reproduced, with permission, from [24]. Abbreviations: $\mathrm{N}$, neo tendon; T, host tendon. 
Table 1. Interplay of Biophysical and Biochemical Properties in Gradient Biomaterials

\begin{tabular}{|c|c|c|c|c|c|}
\hline Feature & Strategy & Technique & Effects on biological functions & Interplay with other features & Refs \\
\hline \multicolumn{6}{|l|}{ Biophysical } \\
\hline \multirow[t]{2}{*}{$\begin{array}{l}\text { Architecture/ } \\
\text { geometry }\end{array}$} & $\begin{array}{l}\text { Multiphasic } \\
\text { scaffolds }\end{array}$ & $\begin{array}{l}\text { Directional freezing and } \\
\text { freeze-drying }\end{array}$ & \multirow{2}{*}{$\begin{array}{l}\text { - Macro/micro/nano interconnected } \\
\text { porosity favors osteogenesis } \\
\text { - Constrained areas promote } \\
\text { chondrogenesis } \\
\text { - Pore shape controls stem cell } \\
\text { differentiation toward chondrogenic (cubic) } \\
\text { or osteogenic (cylindrical) phenotypes } \\
\text { - Aligned 3D geometry induces cell } \\
\text { alignment }\end{array}$} & \multirow{2}{*}{$\begin{array}{l}\text { Dimension and pore shape } \\
\text { gradients induce gradients } \\
\text { of oxygen concentrations }\end{array}$} & \multirow[t]{2}{*}[68,82,83]{} \\
\hline & $\begin{array}{l}\text { Gradient } \\
\text { scaffolds and } \\
\text { hydrogels }\end{array}$ & $\begin{array}{l}\text { Electrospinning; 3D } \\
\text { printing }\end{array}$ & & & \\
\hline \multirow[t]{2}{*}{$\begin{array}{l}\text { Surface } \\
\text { topography }\end{array}$} & $\begin{array}{l}\text { Fiber-based } \\
\text { biomaterials }\end{array}$ & $\begin{array}{l}\text { Electrospinning; } \\
\text { wet spinning }\end{array}$ & \multirow{2}{*}{$\begin{array}{l}\text { - Disordered/random topography } \\
\text { supports osteogenesis } \\
\text { - Greater topographical depth (100 nm) } \\
\text { promotes osteogenesis } \\
\text { - Anisotropic alignment promotes } \\
\text { tenogenesis }\end{array}$} & \multirow{2}{*}{$\begin{array}{l}\text { Organization of topography } \\
\text { alters mechanical } \\
\text { properties }\end{array}$} & \multirow[t]{2}{*}[84]{} \\
\hline & $\begin{array}{l}\text { Patterning } \\
\text { techniques }\end{array}$ & Soft lithography & & & \\
\hline \multirow[t]{2}{*}{$\begin{array}{l}\text { Mechanical } \\
\text { properties }\end{array}$} & $\begin{array}{l}\text { Hydrogel } \\
\text { gradients }\end{array}$ & & \multirow{2}{*}{$\begin{array}{l}\text { - Higher substrate stiffness (>60 kPa) and } \\
\text { elastic moduli induces osteogenesis } \\
\text { - Intermediate substrate stiffness results in } \\
\text { the chondrogenic commitment of stem } \\
\text { cells ( } 30-50 \mathrm{kPa}) \\
\text { - Textile assembling of aligned nanofibers } \\
\text { improves biomechanical performance and } \\
\text { enhances tenogenic differentiation }\end{array}$} & & \multirow[t]{2}{*}[37-40,43,81]{} \\
\hline & $\begin{array}{l}\text { Fiber-based } \\
\text { biomaterials }\end{array}$ & $\begin{array}{l}\text { Electrospinning; } \\
\text { wet spinning; } \\
\text { textile assembling }\end{array}$ & & & \\
\hline \multicolumn{6}{|l|}{ Biochemical } \\
\hline \multirow[t]{2}{*}{ Oxygen } & \multirow[t]{2}{*}{$\begin{array}{l}\text { Hypoxia } \\
\text { conditioning }\end{array}$} & Hypoxic culture & $\begin{array}{l}\text { - Enhanced differentiation potential toward } \\
\text { tenogenic and chondrogenic lineages } \\
\text { - Reduced osteogenic differentiation } \\
\text { commitment of stem cells } \\
\text { - Improve interface integration }\end{array}$ & & {$[65,85]$} \\
\hline & & Chemical induction & $\begin{array}{l}\text { - Gradient of oxygen delivery to control } \\
\text { stem cells differentiation }\end{array}$ & & \\
\hline \multirow[t]{2}{*}{ Composition } & $\begin{array}{l}\text { Growth factors } \\
\text { and other } \\
\text { biomolecules }\end{array}$ & $\begin{array}{l}\text { Click reactions; } \\
\text { tethering/immobilization; } \\
\text { encapsulation; } \\
\text { electrostatic reaction }\end{array}$ & $\begin{array}{l}\text { - Osteoinductive growth factors like } \\
\text { BMP-2 and inorganic elements benefit } \\
\text { osteogenesis } \\
\text { - Growth factors like TGFß3 and BMP-2 } \\
\text { induce endochondral ossification } \\
\text { - TGF } \beta 1 \text { and } 3 \text { induce chondrogenic and } \\
\text { hypertrophic chondrogenic commitment } \\
\text { of stem cells, respectively } \\
\text { - Compositional gradients enable a spatial } \\
\text { control over stem cell differentiation }\end{array}$ & & {$[12,75]$} \\
\hline & Biomineralization & $\begin{array}{l}\text { Crosslinking densities of } \\
\text { polydopamine coatings }\end{array}$ & $\begin{array}{l}\text { - Spatial control over mineral deposition } \\
\text { and control over stem cells differentiation }\end{array}$ & $\begin{array}{l}\text { Biomineralization alters } \\
\text { mechanical properties } \\
\text { (increased stiffness) }\end{array}$ & {$[73,74]$} \\
\hline Gene delivery & $\begin{array}{l}\text { Growth factors } \\
\text { and transcription } \\
\text { factors }\end{array}$ & $\begin{array}{l}\text { Cells } \\
\text { scaffolds }\end{array}$ & $\begin{array}{l}\text { - Plasmid, adenovirus and retrovirus, } \\
\text { baculovirus as vectors for delivery } \\
\text { - Trio-co-transduction of SOX-5, -6, and } \\
\text {-9 combined with RUNX2 enable } \\
\text { osteochondral defect regeneration } \\
\text { - Transfection with FGF-2, GDF-5, and } \\
\text { BMPs benefits chondrogenesis and endo- } \\
\text { chondral ossification }\end{array}$ & & {$[86-89]$} \\
\hline
\end{tabular}

hypoxic culture. Indeed, preconditioning $\left(5 \% \mathrm{O}_{2}\right)$ a 3D bioprinted stratified construct carrying human adipose-derived stem cells reduced uncontrolled differentiation by inhibiting spontaneous calcification and promoted interface integration in vivo [67]. Nonetheless, recreating hypoxic 
niches through culture conditions limits the maintenance of this biomimetic feature to in vitro settings. Strikingly, a minute control over physical features of produced scaffolds has been shown to have impact on oxygen supply. Through variations in pore size (higher to smaller), oxygen diffusion will decrease, resulting in the recreation of a gradient of hypoxic environments within a single structure. This enables a control over stem cell differentiation toward osteogenic and chondrogenic lineages [68].

The role of oxygen tension is frequently disregarded in tissue engineering strategies, but it has been increasingly recognized as a critical biochemical parameter to address in soft-tohard tissue interface regeneration. Novel approaches deploying a control over oxygen gradients are likely to provide a strategic management of multidifferentiation of a single stem cell source.

\section{Growth Factors and Biofunctionalization Strategies}

Musculoskeletal interface tissue repair relies on the use of growth factors to elicit a desired phenotypic response from a host tissue or when co-delivered with cells, through localized and controlled multifunctional delivery systems. Interestingly, growth factors involved in growth plate development have been elucidated as possible targets for both tendon-to-bone and osteochondral tissue regeneration, particularly bone morphogenetic proteins and transforming growth factor beta superfamily [69-71].

Biofunctionalization of biomaterial surfaces have demonstrated good results toward the induction of different phenotypes in single structures. Multifunctional gradients using controllable and reversed click reactions [72] or polymerization of dopamine [73,74] have been explored for tendon-bone interface regeneration. Resulting available groups provide accessibility for biomolecule immobilization and biomineralization [74], allowing a gradual differentiation of stem cells (Figure 3C). Comparably, strategies aimed at the delivery of growth factors for osteochondral unit regeneration have focused on bulk phase delivery, where the release of bioactive factors is dependent on the interaction between the growth factor and the matrix, either by tethering/immobilization [75] or encapsulation [12], resulting in the chondrogenic and osteogenic differentiation of stem cells in single units. In this regard, gradients of growth factors and rate of release have been demonstrated to affect tissue formation. Even though immobilization of growth factors has shown satisfactory results in in vitro applications, this strategy faces some challenges in vivo, such as ion exchange with physiological fluids. Therefore, the undefined and negative cross-effects in in vivo defect studies suggest that these methodologies still require a fine-tuning. Effectively, the need for an 'ideal' spatial and temporal delivery of growth factors in order to potentiate their highest therapeutic efficacy in future tissue engineered approaches is well acknowledged.

\section{Concluding Remarks and Future Perspectives}

Soft-to-hard tissue interfaces have primary mechanical roles. Thus, tissue engineering dedicates a considerable effort toward recapitulating these structures through biomaterial design. Nonetheless, the characteristic complexity of interfacial tissues requires integrative tissue engineering approaches that combine a set of tools (biological, biophysical, and biochemical) toward guiding native or transplanted cells.

Over the years, advances in biotechnological tools have refined TERM strategies. The development of adequate constructs for soft-to-hard tissue interface regeneration is challenged by limited knowledge of the biology of these multitissue transitions.
Outstanding Questions

What are the most critical parameters to control while engineering soft-to-hard tissue physiological environments?

How can such small sized multitissue transitions be precisely engineered?

Can a soft-to-hard tissue engineered construct be produced in clinically translatable setups?

With which resolution can tissuespecific microenvironments be emulated regarding the spatiotemporal dynamics of cellular processes? 


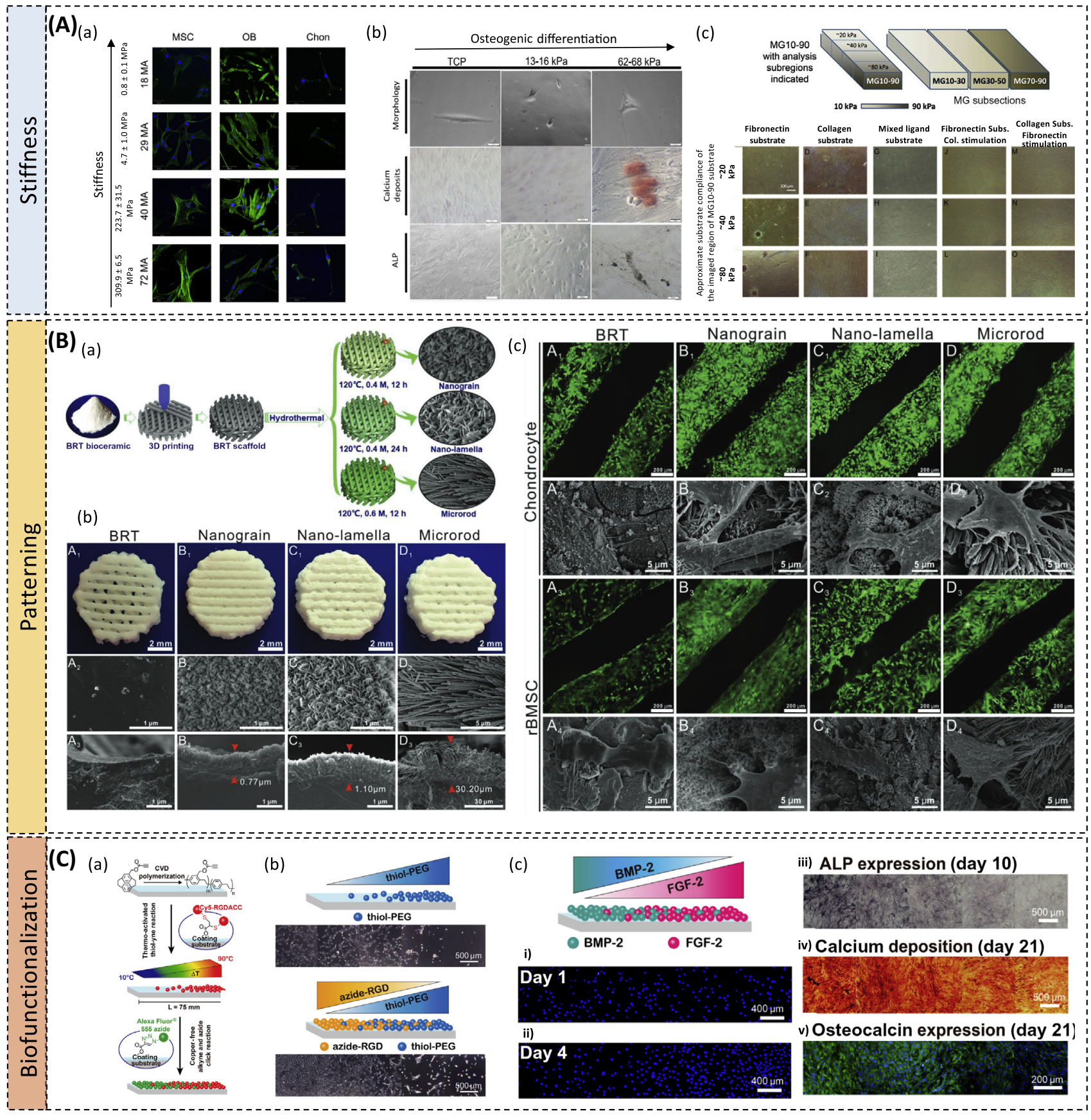

Trends in Biotechnology

Figure 3. Examples of Biophysical Cues to Control Stem Cell Fate for Tendon-to-Bone and Osteochondral Tissue Engineering and Regeneration. As elastic modulus and toughness vary between distinct tissues such as cartilage, tendon/ligament, and bone, a variation between stiffer and softer surfaces has been proven to control stem cell differentiation (A). The stiffness of different methyl acrylate/methyl methacrylate (MA/MMA) polymer surfaces without major changes in surface chemistry were used to evaluate human mesenchymal stem cells (MSC) commitment toward osteoblastic (OB) and chondrogenic (Chon) lineages. Simply by increasing surface stiffness, the tunability of cytoskeletal structural organization was demonstrated (a). Round OBs were found in stiffer substrates (>10 MPa), Chon presented a similar morphology with long extensions and few points of contact, and MSCs presented longer cytoskeletal arrangements in less stiff surfaces ( $<10 \mathrm{MPa})$. Reproduced, with permission, from [38]. The same effect was demonstrated assessing MSC response to tunable polyacrylamide hydrogels coated with fibronectin with increasing stiffness. After 1 week, cells seeded in stiffer substrates (62-68 kPa) were found to produce calcium nodules (Alizarin red-positive) and express more alkaline phosphatase (ALP-stained crystals), even 
Taking advantage of the body's self-healing ability, advanced cell therapies have been increasingly explored. Cell injections rely on taking cells out of their 'comfort zone' and leaving them to face a very harsh environment that is the injury site, leading to cell loss and poor cell engraftment. Although with disadvantages associated with in vitro cellular expansion and extensive manipulation, advanced cell therapies, either based on scaffold-free cell delivery technologies or on cellular actuation through mechanical/magnetic forces, hold potential to change the clinical landscape. Indeed, biotechnological advances enable the generation of regeneration-competent cellular phenotypes and tissue-specific living patches aimed at shifting the profibrotic healing toward a proregenerative environment, which is of particular importance in the case of nonhealing and chronic injuries.

Furthermore, the combination of cell therapies with a support matrix is undoubtedly important, but the reduced size of soft-to-hard tissue insertions also defies the design of gradient biomaterials. Alternative biomaterial designs may focus on aligned-random fibrous scaffolds assembled through textile techniques to guide different cellular phenotypes within the two distinct regions and rely on the cellular crosstalk to recreate an interfacial phenotype when the two cell types merge. Although particularly unpredictable, evidence has been supporting the role of cellular communication in recreating tissue-specific zones. However, future research could focus on improving the resolution of material processing techniques to achieve a better spatial control over physical and biochemical properties and, consequently, cell fate determination.

High-throughput screening technologies have elucidated cellular responses to changes in biophysical and biochemical properties, both at the single cell level and on long-range cell behaviors (see Outstanding Questions). Combining these transcriptomics and proteomics insights with high-resolution fabrication techniques may help to develop more physiologically representative tissue engineered products.

An important aspect of future advanced tissue engineering therapies for soft-to-hard interface regeneration is the establishment of adequate regimes of mechanical stimulation to promote proregenerative cellular responses. For instance, walking steps stimulate soft-to-hard musculoskeletal systems at $1.5 \mathrm{~Hz}$ frequency, under $30.6 \mathrm{~kg} / \mathrm{m}^{2}$ according to the average walking speed and body mass index. To replicate this scenario, dynamic cell culture systems, as bioreactors, have been refined. However, a spatial control over vascularization and innervation in distinct but integrated microtissues is still a main challenge in the field, due to a lack of simultaneous control over biochemical (e.g., growth factors and oxygen tension) and mechanical cues. This way already reported mechanical and biochemical stimulating bioreactors should be combined in a single concept as a means to stimulate distinct microenvironments, allowing a biomimetic regenerative response in soft-hard interfaced tissues.

compared with substrates such as tissue culture plastic (TCP) (b). Reproduced, with permission, from [81]. Materials stiffness can also influence tendon differentiation. Polyacrylamide hydrogels with mechanical gradient comprising a moderately rigid collagen type I substrate ( $\sim 30-50 \mathrm{kPa})$ was found to induce MSC differentiation into tenogenic lineage, while MSCs differentiate into osteogenic cells on more rigid substrates (70-90 kPa) (c). Reproduced, with permission, from [40]. (B) Surface patterning of 3D scaffolds with different morphological micro/nanostructured surface has demonstrated potential use for cartilage and subchondral bone application (a). Pure bredigite, a bioactive composite made of silica, magnesium, and calcium, with good bioactivity, biodegradability, and mechanical properties, was used to produce the 3D scaffolds. Structured surfaces distinctly facilitated the spread and differentiation of chondrocytes, regulated cell morphology, and promoted osteogenic differentiation of rBMSCs ( $b, c)$. Reproduced, with permission, from [46]. (C) Surface modification by dual reverse click reactions producing a continuous and gradient biofunctionalization to control stem cell differentiation for tendon-to-bone regeneration. (a) Seeding with 3T3 fibroblasts on surfaces containing (a) only PEG immobilized to the first gradient and (b) two gradients of PEG and RGD contrary gradients, demonstrated a cell-dependent behavior for PEG. After 21 days with human adipose tissue-derived stem cells (ASCs) in functionalized surfaces with BMP2 and FGF-2, a gradient was observed of (iii) ALP expression, (iv) calcium deposition, and (v) osteocalcin expression, showing the osteogenic commitment of hASCs in a continuous and gradient manner. Reproduced, with permission, from [72]. Abbreviations: rBMSC: rat bone marrow mesenchymal stem cells. 


\section{Acknowledgments}

The authors acknowledge the financial support from the European Union Framework Programme for Research and Innovation HORIZON2020 (TEAMING Grant agreement, No 739572 - The Discoveries CTR), the ERC Grant CoG MagTendon (nr 772817), Fundação para a Ciência e a Tecnologia (FCT) for the PhD grant of I.C. (PD/BD/128088/2016), and the Project NORTE-01-0145-FEDER-000021 through the European Regional Development Fund (ERDF).

\section{References}

1. Goldring, S.R. and Goldring, M.B. (2016) Changes in the osteochondral unit during osteoarthritis: structure, function and cartilage-bone crosstalk. Nat. Rev. Rheumatol. 12, 632

2. Rossetti, L. et al. (2017) The microstructure and micromechanics of the tendon-bone insertion. Nat. Mater. 16, 664

3. Briggs, A.M. et al. (2016) Musculoskeletal health conditions represent a global threat to healthy aging: a report for the 2015 World Health Organization World Report on Ageing and Health. Gerontologist 56, S243-S255

4. Sambandam, S.N. et al. (2015) Rotator cuff tears: an evidence based approach. World J. Orthop. 6, 902-918

5. Chen, J. et al. (2009) Scaffolds for tendon and ligament repair: review of the efficacy of commercial products. Expert Rev. Med. Devices 6, 61-73

6. Bicho, D. et al. (2018) Commercial products for osteochondral tissue repair and regeneration. Adv. Exp. Med. Biol. 1058, 415-428

7. Li, Y. et al. (2017) The horizon of materiobiology: a perspective on material-guided cell behaviors and tissue engineering. Chem. Rev. 117, 4376-4421

8. Kim, B.S. et al. (2014) Human collagen-based multilayer scaf folds for tendon-to-bone interface tissue engineering. J. Biomed. Mater. Res. A 102, 4044-4054

9. Huang, G.X. et al. (2015) Modeling and validation of multilayer poly(lactide-co-glycolide) scaffolds for in vitro directed differentiation of juxtaposed cartilage and bone. Tissue Eng. Part A 21 , 2228-2240

10. Perikamana, M. et al. (2018) Harnessing biochemical and structural cues for tenogenic differentiation of adipose derived stem cells (ADSCs) and development of an in vitro tissue interface mimicking tendon-bone insertion graft. Biomaterials 165, 79-93

11. Tellado, S.F et al. (2018) Heparin functionalization increases retention of TGF- $\beta 2$ and GDF5 on biphasic silk fibroin scaffolds for tendon/ligament-to-bone tissue engineering. Acta Biomater. 72 150-166

12. Gao, F. et al. (2018) Direct 3D printing of high strength biohybrid gradient hydrogel scaffolds for efficient repair of osteochondra defect. Adv. Funct. Mater. 28, 1706644

13. Levingstone, T.J. et al. (2016) Multi-layered collagen-based scaffolds for osteochondral defect repair in rabbits. Acta Biomater. 32, 149-160

14. Chen, J. et al. (2011) Simultaneous regeneration of articular cartilage and subchondral bone in vivo using MSCs induced by a spatially controlled gene delivery system in bilayered integrated scaffolds. Biomaterials 32, 4793-4805

15. Bunpetch, V. et al. (2019) Silicate-based bioceramic scaffolds for dual-lineage regeneration of osteochondral defect. Biomaterials 192, 323-333

16. Zhu, Y. et al. (2019) An injectable continuous stratified structurally and functionally biomimetic construct for enhancing osteochondral regeneration. Biomaterials 192, 149-158

17. Canadas, R.F. et al. (2018) Biochemical gradients to generate 3D heterotypic-like tissues with isotropic and anisotropic architectures. Adv. Funct. Mater. 28, 1804148

18. Radhakrishnan, J. et al. (2018) Gradient nano-engineered in situ forming composite hydrogel for osteochondral regeneration. Biomaterials 162, 82-98

19. Loebel, C. and Burdick, J.A. (2018) Engineering stem and stromal cell therapies for musculoskeletal tissue repair. Cell Stem Cell 22, 325-339

20. Yamato, M. and Okano, T. (2004) Cell sheet engineering. Mater Today $7,42-47$

21. Ebihara, G. et al. (2012) Cartilage repair in transplanted scaffoldfree chondrocyte sheets using a minipig model. Biomaterials 33 , 3846-3851
22. Ito, S. et al. (2012) Repair of articular cartilage defect with layered chondrocyte sheets and cultured synovial cells. Biomaterials 33 5278-5286

23. Wang, F. et al. (2018) Scaffold-free cartilage cell shee combined with bone-phase BMSCs-scaffold regenerate osteochondral construct in mini-pig model. Am. J. Transl. Res. 10, 2997-3010

24. Zhang, C. et al. (2018) Histone deacetylase inhibitor treated cel sheet from mouse tendon stem/progenitor cells promotes tendon repair. Biomaterials 172, 66-82

25. Liu, Q. et al. (2019) Engineered tendon-fibrocartilage-bone composite and bone marrow-derived mesenchymal stem cell sheet augmentation promotes rotator cuff healing in a non-weightbearing canine model. Biomaterials 192, 189-198

26. Takahashi, H. et al. (2011) Anisotropic cell sheets for constructing three-dimensional tissue with well-organized cell orientation. Biomaterials 32, 8830-8838

27. Liu, C. et al. (2017) Light-induced cell alignment and harvest for anisotropic cell sheet technology. ACS Appl. Mater. Interfaces 9 36513-36524

28. Gonçalves, A.l. et al. (2017) Tissue-engineered magnetic cell sheet patches for advanced strategies in tendon regeneration. Acta Biomater. 63, 110-122

29. Sant, S. et al. (2017) Self-assembled hydrogel fiber bundles from oppositely charged polyelectrolytes mimic micro-/nanoscale hierarchy of collagen. Adv. Funct. Mater. 27, 1606273

30. Calejo, I. et al. (2019) A textile platform using continuous aligned and textured composite microfibers to engineer tendon. $A d v$. Healthc. Mater. https://doi.org/10.1002/adhm.201900200 Published online June 13, 2019

31. Li, L. et al. (2019) 3D molecularly functionalized cell-free biomimetic scaffolds for osteochondral regeneration. Adv. Funct. Mater. 29, 1807356

32. Mendes, B.B. et al. (2018) Human-based fibrillar nanocomposite hydrogels as bioinstructive matrices to tune stem cell behavior. Nanoscale 10, 17388-17401

33. Araújo-Custódio, S. et al. (2019) Injectable and magnetic responsive hydrogels with bioinspired ordered structures. ACS Biomater. Sci. Eng. 5, 1392-1404

34. Baker, B.M. et al. (2015) Cell-mediated fibre recruitment drives extracellular matrix mechanosensing in engineered fibrillar microenvironments. Nat. Mater. 14, 1262-1268

35. Felsenthal, N. and Zelzer, E. (2017) Mechanical regulation of musculoskeletal system development. Development 144, 4271-4283

36. Engler, A. et al. (2006) Matrix elasticity directs stem cell lineage specification. Cell 126, 677-689

37. Wu, Y. et al. (2017) The combined effect of substrate stiffness and surface topography on chondrogenic differentiation of mesenchymal stem cells. Tissue Eng. Part A 23, 43-54

38. Olivares-Navarrete, R. et al. (2017) Substrate stiffness controls osteoblastic and chondrocytic differentiation of mesenchymal stem cells without exogenous stimuli. PLoS One 12, e0170312

39. Sharma, R.I. and Snedeker, J.G. (2010) Biochemical and biomechanical gradients for directed bone marrow stromal cell differentiation toward tendon and bone. Biomaterials 31, 7695-7704

40. Sharma, R.I. and Snedeker, J.G. (2012) Paracrine interactions between mesenchymal stem cells affect substrate driven differentiation toward tendon and bone phenotypes. PLoS One 7, e31504

41. Liu, W. et al. (2014) Nanofiber scaffolds with gradients in mineral content for spatial control of osteogenesis. ACS Appl. Mater. Interfaces 6, 2842-2849

42. Liu, S.Q. et al. (2010) Biomimetic hydrogels for chondrogenic differentiation of human mesenchymal stem cells to neocartilage. Biomaterials 31, 7298-7307 
43. Wang, T. et al. (2016) Effects of hydrogel stiffness and extracellular compositions on modulating cartilage regeneration by mixed populations of stem cells and chondrocytes in vivo. Tissue Eng. Part A 22, 1348-1356

44. Shi, Y. et al. (2017) Microgrooved topographical surface directs tenogenic lineage specific differentiation of mouse tendon derived stem cells. Biomed. Mater. 12, 015013

45. Mahapatra, C. et al. (2019) Differential chondro- and osteostimulation in three-dimensional porous scaffolds with different topological surfaces provides a design strategy for biphasic osteochondral engineering. J. Tissue Eng. 10, 2041731419826433

46. Deng, C. et al. (2018) Micro/nanometer-structured scaffolds for regeneration of both cartilage and subchondral bone. Adv. Funct. Mater. 29, 1806068

47. Nowlin, J. et al. (2018) Engineering the hard-soft tissue interface with random-to-aligned nanofiber scaffolds. Nanobiomedicine (Rij) 5, 1849543518803538

48. Darnell, M. et al. (2018) Material microenvironmental properties couple to induce distinct transcriptional programs in mammalian stem cells. Proc. Natl. Acad. Sci. U. S. A. 115, E8368-E8377

49. Dalby, M.J. et al. (2014) Harnessing nanotopography and integrin-matrix interactions to influence stem cell fate. Nat. Mater. 13, 558

50. Wen, J.H. et al. (2014) Interplay of matrix stiffness and protein tethering in stem cell differentiation. Nat. Mater. 13, 979

51. Chaudhuri, O. et al. (2014) Extracellular matrix stiffness and composition jointly regulate the induction of malignant phenotypes in mammary epithelium. Nat. Mater. 13, 970

52. Darnell, M. et al. (2018) RNA-seq reveals diverse effects of substrate stiffness on mesenchymal stem cells. Biomaterials 181, 182-188

53. Yang, C. et al. (2014) Mechanical memory and dosing influence stem cell fate. Nat. Mater. 13, 645-652

54. Thorpe, S.D. et al. (2013) Modulating gradients in regulatory signals within mesenchymal stem cell seeded hydrogels: a novel strategy to engineer zonal articular cartilage. PLoS One 8, e60764

55. Liu, Q. et al. (2018) Novel engineered tendon-fibrocartilage-bone composite with cyclic tension for rotator cuff repair. J. Tissue Eng. Regen. Med. 12, 1690-1701

56. Ni, M. et al. (2013) Engineered scaffold-free tendon tissue produced by tendon-derived stem cells. Biomaterials 34 2024-2037

57. Lui, P.P.Y. et al. (2016) Transplantation of tendon-derived stem cells pre-treated with connective tissue growth factor and ascorbic acid in vitro promoted better tendon repair in a patellar tendon window injury rat model. Cytotherapy 18, 99-112

58. Hsieh, C.-F. et al. (2018) In vitro comparison of 2D-cell culture and 3D-cell sheets of scleraxis-programmed bone marrow derived mesenchymal stem cells to primary tendon stem/ progenitor cells for tendon repair. Int. J. Mol. Sci. 19, 2272

59. Shimizu, R. et al. (2015) Repair mechanism of osteochondral defect promoted by bioengineered chondrocyte sheet. Tissue Eng. Part A 21, 1131-1141

60. Koh, M.Y. and Powis, G. (2012) Passing the baton: the HIF switch. Trends Biochem. Sci. 37, 364-372

61. Zhang, J. and Wang, J.H. (2013) Human tendon stem cells better maintain their stemness in hypoxic culture conditions. PLoS One 8, e61424

62. Yu, Y. et al. (2017) Effect of hypoxia on self-renewal capacity and differentiation in human tendon-derived stem cells. Med. Sci. Monit. 23, 1334-1339

63. Kang, H. et al. (2017) Osteoblast hypoxia-inducible factor-1alpha pathway activation restrains osteoclastogenesis via the interleukin33-microRNA-34a-Notch1 pathway. Front. Immunol. 8, 1312

64. Marenzana, M. and Arnett, T.R. (2013) The key role of the blood supply to bone. Bone Res. 1, 203-215

65. Stiers, P.J. et al. (2018) Inhibition of the oxygen sensor PHD2 enhances tissue-engineered endochondral bone formation. J. Bone Miner. Res. 34, 333-348

66. Huang, X. et al. (2018) Promoted chondrogenesis of cocultured chondrocytes and mesenchymal stem cells under hypoxia using in-situ forming degradable hydrogel scaffolds. Biomacromolecules 19, 94-102
67. Wang, Y. et al. (2017) Effects of hydroxyapatite and hypoxia on chondrogenesis and hypertrophy in 3D bioprinted ADMSC laden constructs. Biomater. Sci. Eng. 3, 826-835

68. Di Luca, A. et al. (2016) Tuning cell differentiation into a 3D scaffold presenting a pore shape gradient for osteochondral regeneration. Adv. Healthc. Mater. 5, 1753-1763

69. Liao, J. et al. (2014) Sox9 potentiates BMP2-induced chondrogenic differentiation and inhibits BMP2-induced osteogenic differentiation. PLoS One 9, e89025

70. Mendes, L.F. et al. (2018) Advancing osteochondral tissue engineering: bone morphogenetic protein, transforming growth factor, and fibroblast growth factor signaling drive ordered differentiation of periosteal cells resulting in stable cartilage and bone formation in vivo. Stem Cell Res Ther 9, 42

71. Zhou, N. et al. (2016) BMP2 induces chondrogenic differentiation, osteogenic differentiation and endochondral ossification in stem cells. Cell Tissue Res. 366, 101-111

72. Guan, Z.-Y. et al. (2016) Multifunctional and continuous gradients of biointerfaces based on dual reverse click reactions. ACS Appl. Mater. Interfaces 8, 13812-13818

73. Perikamana, S.K.M. et al. (2017) Graded functionalization of biomaterial surfaces using mussel-inspired adhesive coating of polydopamine. Colloids Surf. B Biointerface 159, 546-556

74. Madhurakkat Perikamana, S.K. et al. (2017) Oxygen-dependent generation of a graded polydopamine coating on nanofibrous materials for controlling stem cell functions. J. Mater. Chem. B 5, 8865-8878

75. Stüdle, C. et al. (2018) Spatially confined induction of endochondral ossification by functionalized hydrogels for ectopic engineering of osteochondral tissues. Biomaterials 171, 219-229

76. Provenzano, P. and Vanderby, R. (2006) Collagen fibril morphology and organization- implications for force transmission in ligament and tendon. Matrix Biol. 25, 71-84

77. McNally, E.A. et al. (2012) A model for the ultrastructure of bone based on electron microscopy of ion-milled sections. PLoS One 7, e29258.

78. Fujie, H. and Imade, K. (2015) Effects of low tangential permeability in the superficial layer on the frictional property of articular cartilage. Biosurf. Biotribol. 1, 124-129

79. Hunziker, E.B. et al. (2002) Quantitative structural organization of normal adult human articular cartilage. Osteoarthr. Cartil. 10, 564-572

80. Rieppo, J. et al. (2009) Changes in spatial collagen content and collagen network architecture in porcine articular cartilage during growth and maturation. Osteoarthr. Cartil. 17, 448-455

81. Sun, M. et al. (2018) Extracellular matrix stiffness controls osteogenic differentiation of mesenchymal stem cells mediated by integrin alpha5. Stem Cell Res. Ther. 9, 52

82. Park, K.M. and Gerecht, S. (2014) Hypoxia-inducible hydrogels. Nat. Commun. 5, 4075

83. Ferlin, K.M. et al. (2016) Influence of 3D printed porous architecture on mesenchymal stem cell enrichment and differentiation. Acta Biomater. 32, 161-169

84. Zouani, O.F. et al. (2012) Altered nanofeature size dictates stem cell differentiation. J. Cell Sci. 125, 1217

85. Farris, A.L. et al. (2016) Oxygen delivering biomaterials for tissue engineering. J. Mater. Chem. B 4, 3422-3432

86. Lee, J.-M. and Im, G.-I. (2012) SOX trio-co-transduced adipose stem cells in fibrin gel to enhance cartilage repair and delay the progression of osteoarthritis in the rat. Biomaterials 33 , 2016-2024

87. Needham, C.J. et al. (2014) Osteochondral tissue regeneration through polymeric delivery of DNA encoding for the SOX trio and RUNX2. Acta Biomater. 10, 4103-4112

88. An, C. et al. (2010) IGF-1 and BMP-2 induces differentiation of adipose-derived mesenchymal stem cells into chondrocyteslike cells. Ann. Biomed. Eng. 38, 1647-1654

89. Cucchiarini, M. et al. (2011) Metabolic activities and chondrogenic differentiation of human mesenchymal stem cells following recombinant adeno-associated virus-mediated gene transfer and overexpression of fibroblast growth factor 2. Tissue Eng. Part A 17, 1921-1933 